\title{
RONCUS (PARABLOTHRUS) SETOSUS \\ N. SP., OTRO CASO DE \\ «NÉOCHÉTOTAXIE MAJORANTE \\ PROSOMATIQUE» (HEURTAULT) \\ EN LOS PSEUDOSCORPIONES \\ NEOBISIIDAE.
}

Por Juan Antonio Zaragoza ${ }^{1}$

\begin{abstract}
A new species of Pseudoscorpion belonging to the family Neobisiidae is described: Roncus (Parablothrus) setosus. Easily distinguishable of any other species of the genus by the extraordinary number of setae on the carapace and tergites, representing another case of «néochétotaxie majorante prosomatique» J. HEURTAULT 1980, this being confirmed by the chaetotaxy found in tritonymph and deutonymph.
\end{abstract}

\section{AGRADECIMIENTOS}

Quiero testimoniar mi agradecimiento a las siguientes personas de la Facultad de Ciencias de la Universidad de Alicante, por el apoyo que en todo momento me han brindado y haber puesto a mi disposición las instalaciones de sus respectivos departamentos: Drs. Antonio Escarré y Eduardo Seva del Departamento de Biología, Dr. Carlos Auernheimer y D. José A. Pina del Departamento de Geología.

Un especial agradecimiento al Dr. Wolker Mahnert, de Ginebra, por sus útiles orientaciones y haberse prestado amablemente a la revisión del presente trabajo. 


\section{INTRODUCCIÓN}

En 1980, J. HEURTAULT utiliza el término «néochétotaxis majorante prosomatique» para la descripción de dos nuevas especies de pseudoscorpiones del género Neobisium: $N$. pyrenaicum y $N$. mahner$t i$, que presentan sobre el carapacho un número de sedas anormalmente alto. En el mismo trabajo, el citado autor efectúa previamente el estudio de la fórmula prosomática de varias especies de Neobisiidae que muestran una importante variabilidad, afectando sobre todo a la parte posterior del prosoma; viniendo a concluir que desde los adultos de algunas especies se hace imposible el establecimiento de una fórmula de base, y que ésta sólo es factible desde la deutoninfa, donde el número de sedas es estable; de aquí la importancia del estudio del desarrollo postembrionario en aquellas especies sometidas a variabilidad. En N. pyrenaicum y $N$. mahnerti, y también en Pachyolpium atlanticum, MAHNERT 1981, la adición además en los adultos, desde la fórmula de base, de múltiples sedas concirniendo a todo el prosoma, hace hablar en estos casos de «néochétotaxie majorante» como una especialización evolutiva.

En el presente trabajo se describe una nueva especie, perteneciente al género Roncus y representando otro caso de «néochétotaxie majorante», lo que viene confirmado por la quetotaxia hallada en la tritoninfa y deutoninfa.

\section{Roncus (Parablothrus) setosus n. sp.}

Material: Sa Cova des Vells, Tárbena, Alicante, leg. J. A. Zaragoza, 28.11.82: 1 Q (Holotipo), 23.01.83: $1{ }^{\prime}, 1$ tritoninfa, 1 deutoninfa (Paratipos). (Holotipo y paratipos en la colección del autor).

\section{DESCRIPCIÓN DE LOS ADULTOS}

Carapacho (fig. 1) claramente más largo que ancho, con epistoma triangular, puntiagudo; con manchas oculares, una cada lado; con 49 sedas (6-14-19-10), ( $0^{*}: 51$ sedas $\left.=6-13-22-10\right)$ y además con dos grupos de 9 sedas cada uno sobre los bordes inferiores de las caras laterales anteriores del carapacho (fig. 5), estos bordes son visibles dorsalmente.

Sedas de los terguitos: 10-10-11-10-11-13-11-11-11-9 (de ellas 4 se- 


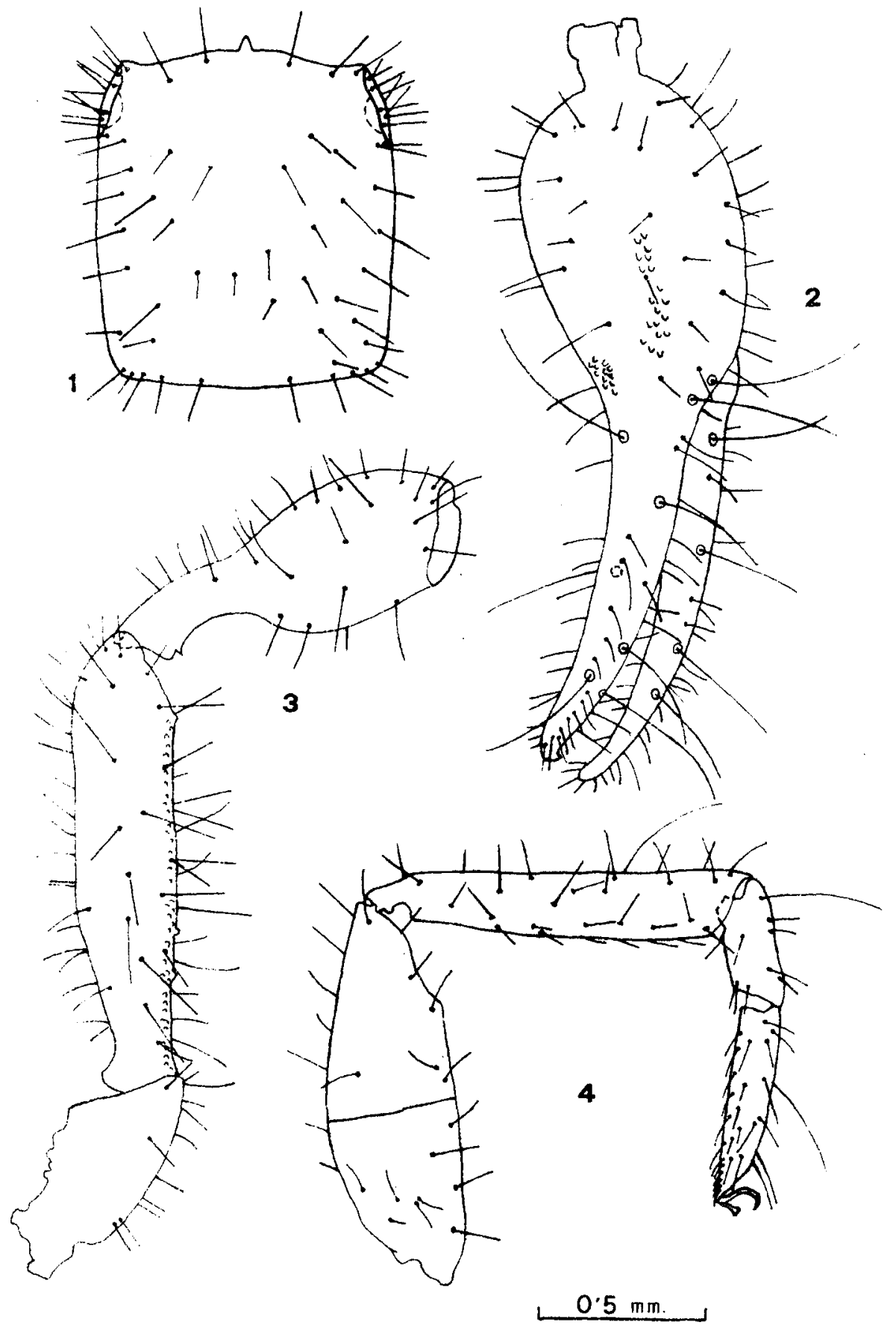

Roncus (Parablothrus) setosus n. sp.

1: Carapacho; 2-3: Pedipalpo izquierdo; 4: Pata IV. 

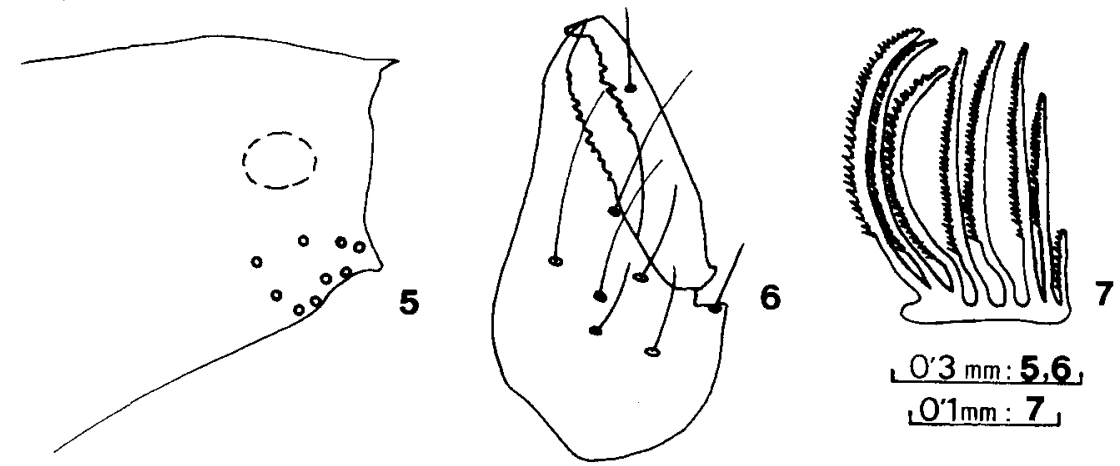

5: Vista lateral anterior del carapacho. Posición de las sedas laterales; 6: Quelícero derecho; 7: Flagelo.

das tactíles), (Ơ : 10-12-11-12-12-12-12-12-12-9), último segmento con 12 sedas (de ellas 6 sedas tactíles); cono anal con 4 sedas.

Quelícero (fig. 6) con 7 sedas en la mano ( $\sigma^{*}$ con 6, falta $a c_{3}$ ). Los dientes del quelícero desgastados, dedo fijo con 14 dientes muy uniformes, dedo móvil con 12 dientes, los dos centrales más ensanchados (mucho más desgastados en el $\sigma^{*}$, sólo pueden distinguirse 6 dientes sobre el dedo móvil y casi imperceptibles en el fijo). La seda subgaleal distal del centro (TS: 0,73 ). Longitud del dedo móvil: $0,46 \mathrm{~mm}$. Serrulla externa con 37 láminas ( $O^{*} 35$ ), serrulla interna con 30 láminas ( $O^{*} 30$ ). Flagelo (fig. 7) típico del género, con 8 sedas bien pectinadas, la próxima a la base mucho más corta que el resto.

Lóbulo de la coxa del pedipalpo con 4 sedas. Coxa del pedipalpo con 8 , coxa I con 6, coxa II con 6, coxa III con 5 y coxa IV con 8 sedas. Pedipalpos (figs. 2-3) con el fémur granulado en su cara interna y con 2-3 protuberancias mayores en la mitad basal; trócanter con un pronunciado tubérculo en su cara ventral (más pequeño en el $\sigma^{*}$ ); fémur con mango, la mitad distal con una anchura uniforme, 4,34 X más largo que ancho ( $\left.0^{\circ} 4,83 \mathrm{X}\right)$; tibia $2,60 \mathrm{X}(3,11 \mathrm{X})$, maza $1,53 \mathrm{X}(1,71 \mathrm{X})$, mano con mango $1,67 \mathrm{X}(2,03 \mathrm{X})$, pinzas con mango 3,60 X (4,08 X), dedo bastante más largo que la mano con mango y de la misma longitud que el fémur, ambos claramente más largos que el carapacho. De- 
do fijo con 80 dientes pequeños y uniformes ( $\sigma^{96}$ ), dedo móvil con 81 (90). Posición de los tricobotrios según figura 2.

Pata I: fémur I 4,10 X más largo que ancho ( $\mathrm{O}^{*} 4 \mathrm{X}$ ), fémur II $3,40 \mathrm{X}(3,31 \mathrm{X})$, tibia $6,18 \mathrm{X}(6,09 \mathrm{X})$, tarso $\mathrm{I} 4,25 \mathrm{X}(3,89 \mathrm{X})$, tarso Il 7,14 X (6,38 X); fémur I 1,37 X más largo que fémur II $(1,36 \mathrm{X})$, tarso II $1,47 \mathrm{X}$ más largo que tarso I $(1,46 \mathrm{X})$.

Pata IV (fig. 4): fémur 2,98 X más largo que ancho $(3 \mathrm{X})$, tibia $5,58 \mathrm{X}(5,80 \mathrm{X})$ con seda tactíl (TS: 0,66$)$, tarso I 2,50 X (3 X) con seda tactíl (TS: 0,14$)$, tarso II 4,62 X (6 X) con seda tactíl (TS: 0,39$)$.

Medidas del cuerpo: en mm.

Longitud del cuerpo: 3,13 ( $\odot$ 3,05).

Carapacho: $1,06-0,89(1,03-0,85)$.

Pedipalpos: fémur $1,39-0,32(1,40-0,29)$, tibia $1,12-0,43(1,18-0,38)$, mango $0,46(0,53)$, mano con mango $1,10-0,66(1,18-0,58)$, mango 0,19 $(0,20)$, dedo $1,37(1,40)$.

Pata I: fémur I 0,70-0,17 (0,72-0,18), fémur II 0,51-0,15 $(0,53-0,16)$, tibia $0,68-0,11(0,67-0,11)$, tarso I $0,34-0,08(0,35-0,09)$, tarso II $0,50-0,07$ $(0,51-0,08)$.

Pata IV: fémur $1,19-0,40(1,14-0,38)$, tibia $1,17-0,20(1,16-0,20)$, tarso I $0,40-0,16(0,36-0,12)$, tarso II $0,60-0,13(0,60-0,10)$.

\section{DESCRIPCIÓN DE LA TRITONINFA PARATIPO}

Carapacho (fig. 9a) un poco más largo que ancho, con epistoma levemente prominente; con manchas oculares; con 38 sedas (4-12-13-9) y además 6 sedas laterales.

Terguitos, del I al X: 8-9-10-11-11-11-11-11-12-12 sedas.

Quelícero con 6 sedas en la mano y 1 seda subgaleal.

Pedipalpos: fémur 3,82 X más largo que ancho, tibia 2,69 X, maza 1,81 X, mano con mango 1,97 X, pinzas con mango 3,80 X. Dedos de la pinza con 10 tricobotrios (fig. 9 b), 7 en el fijo y 3 en el móvil: faltan is $b$ y $s b$; con 61 dientes en cada dedo. 

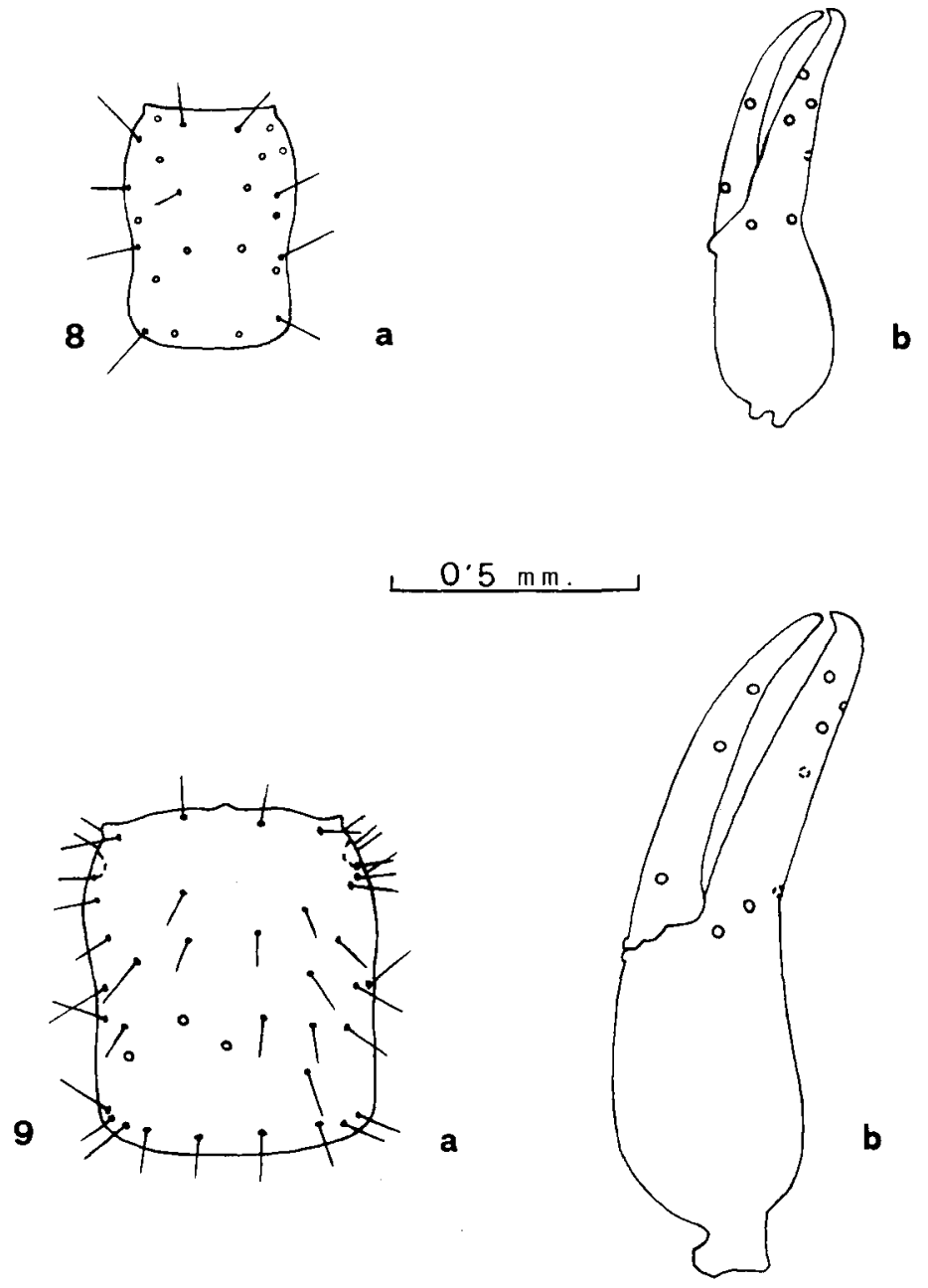

8: Deutoninfa paratipo. a) Carapacho. b) Pinza.

9: Tritoninfa paratipo. a) Carapacho. b) Pinza. 
Medidas del cuerpo: en mm.

Longitud del cuerpo: 1,70 .

Carapacho: $0,72-0,60$.

Pedipalpos: fémur $0,84-0,22$, tibia $0,70-0,26$, mango 0,23 , mano con mango $0,75-0,38$, dedo 0,80 .

\section{DESCRIPCIÓN DE LA DEUTONINFA PARATIPO}

Carapacho (fig. 8 a) claramente más largo que ancho: 1,40 X, sin epistoma; con 24 sedas (4-10-6-4).

Quetotaxia de los terguitos anteriores: 4-5-5-6-6-.

Quelícero con 5 sedas en la mano y 1 seda subgaleal.

Pedipalpos: fémur 3,50 X, tibia 2,56 X, maza 1,69 X, mano con mango $1,92 \mathrm{X}$, pinzas con mango $3,40 \mathrm{X}$. Dedos de la pinza con 8 tricobotrios (fig. 8 b), 6 en el fijo y 2 en el móvil: faltan $e s b$, isb, y $s b$, $t$; dedo fijo con 42 dientes, el móvil con 37 .

Medidas del cuerpo: en mm.

Longitud del cuerpo: 1,24.

Carapacho: $0,49-0,35$.

Pedipalpos: fémur $0,49-0,14$, tibia $0,41-0,16$, mango 0,14 , mano $0,46-0,24$, dedo 0,50 .

\section{DISCUSIÓN}

Especie próxima a Roncus (P.) boneti BEIER 1931 por el epistoma triangular, el gran tubérculo del trocánter y las proporciones y medidas del pedipalpo (el holotipo $\subsetneq$ muestra las proporciones de la su- 
bespecie boneti boneti BEIER 1931, mientras que el paratipo ơ responde a las de boneti tarbenae MAHNERT 1977). Fácilmente distinguible de ésta y de cualquier otra especie del género Roncus por el extraordinario número de sedas sobre el carapacho y los terguitos.

La variabilidad quetotáxica prosomática durante el desarrollo postembrionario (Deutoninfa $\rightarrow$ Tritoninfa $\rightarrow$ Adulto) difiere notablemente de unas especies a otras, pero manteniéndose siempre para una misma especie dentro de un número de sedas más o menos próximo, como en el caso de Roncus lubricus que pese a mostrar una variabilidad importante, aún puede intuirse el esquema de base (D: 4-4-8-6; T: 4/5-5/7-7/8-6; A: 4-5/7-7/8-6); mientras que Roncus setosus viene a inscribirse como un caso similar a Neobisium pyrenaicum (T: 4-9-9-13; A: 4/6-9/13-15/23-9,14), Neobisium mahnerti (A: 4-8-15-12) y Pachyolpium atlanticum (D: 6-4/6-6-3/4-4; T: 6-9-9-6-6; A: $6-10 / 15-10 / 15-9 / 15-6 / 11)$, donde el esquema de base es totalmente modificado por un aumento considerable del número de sedas y se hace irreconocible desde los adultos.

\section{BIBLIOGRAFÍA}

BEIER, M. 1931. Zur Kenntnis der troglobionten Neobisien (Pseudoscorp.). Eos, Madrid, tomo VII, $1^{\circ}: 9-23$.

BEIER, M.: 1963. Ordnung Pseudoscorpionidea (Afterskorpione). Bestimmungsbücher zur Bodenfauna Europas, Berlín, 1-313 pp.

HEURTAULT, J. 1980. La néochétotaxie majorante prosomatique chez les Pseudoscorpions Neobisiidae: Neobisium pyrenaicum et $N$. mahnerti sp. n. C.R. Vè Colloque d'Arachnologie d'expression française, Barcelona, 87-97.

MAHNERT, V. 1977. Spanische Höhlenpseudoskorpione. Miscelánea Zoológica, Barcelona, vol. IV, fasc. 1: 61-104.

MAHNERT, V. y SCHUSTER, R. 1981. Pachyolpium atlanticum n. sp., ein Pseudoskorpion aus der Gezeitenzone der Bermudas: Morphologie und Ökologie (Pseudoscorpiones: Olpiidae). Revue suisse Zool., Genève, tome 88, fasc. 1: 265-273. 